Shingo Maeda • Hiroaki Koga - Shunji Matsunaga

Takuya Numasawa · Katsunori Ikari · Kozo Furushima

Seiko Harata · Jun Takeda • Takashi Sakou

Setsuro Komiya • Ituro Inoue

\title{
Gender-specific haplotype association of collagen $\alpha 2$ (XI) gene in ossification of the posterior longitudinal ligament of the spine
}

Received: September 5, 2000 / Accepted: October 2, 2000

\begin{abstract}
Among Japanese, ossification of the posterior longitudinal ligament of the spine (OPLL) is a leading cause of myelopathy, showing ectopic bone formation in the paravertebral ligament. We have provided genetic evidence that the collagen $\alpha 2$ (XI) (COL11A2) locus of chromosome 6 constitutes susceptibility for OPLL. Five distinct single nucleotide polymorphisms (SNPs), identified in COL11A2, were combined to construct possible haplotypes by the use of a maximum likelihood program. Estimated haplotype frequency was compared in OPLL patients and non-OPLL controls. We report a gender-specific association of the COL11A2 haplotype with OPLL. The frequency of the most commonly observed haplotype was significantly higher in male patients $(P=0.0003)$ compared with controls, but not in female patients $(P=0.21)$. OPLL is predominantly observed in males, with a prevalence ratio of $2: 1$, and our gender-specific associations indicate that genetic factors involving COL11A2 play a specific role in the etiology of OPLL exclusively in males.
\end{abstract}

Key words SNPs $\cdot$ Haplotype $\cdot$ Association study $\cdot$ OPLL · COL11A2

S. Maeda $\cdot$ H. Koga $\cdot$ S. Matsunaga $\cdot$ S. Komiya

Department of Orthopedic Surgery, Faculty of Medicine, Kagoshima University, Kagoshima, Japan

T. Numasawa $\cdot$ K. Ikari $\cdot$ K. Furushima $\cdot$ S. Harata

Department of Orthopedic Surgery, School of Medicine, Hirosaki

University, Hirosaki, Japan

S. Maeda $\cdot$ K. Ikari $\cdot$ K. Furushima $\cdot$ I. Inoue $(\triangle)$

Division of Genetic Diagnosis, The Institute of Medical Science,

The University of Tokyo, 4-6-1 Shirokanedai, Minato-ku,

Tokyo 108-8639, Japan

Tel. +81-3-5449-5765; Fax +81-3-5449-5764

e-mail: ituro@ims.u-tokyo.ac.jp

J. Takeda

Department of Cell Biology, Institute for Molecular and Cellular

Regulation, Gunma University, Maebashi, Japan

T. Sakou

Sakou Clinic, Kagoshima, Japan

\section{Introduction}

Ossification of the posterior longitudinal ligament of the spine (OPLL) is characterized by heterotopic bone formation in spinal ligaments, mainly by an endochondral ossification process. Ossified ligaments compress the spinal cord, and this subsequently leads to various degrees of myelopathy. OPLL is a common disorder among Japanese, as well as in other Asian populations. The incidence of OPLL in Japan is reported to be $1.9 \%-4.3 \%$ of the general population (Matsunaga and Sakou 1997). Despite the fact that OPLL is a late-onset disease, with an average onset age of $\sim 50$ years, genetic determinants play a crucial role in its etiology (Terayama 1989). In the United States and Europe, OPLL is less common, with a reported frequency of $0.01 \%-$ $1.7 \%$. Another ossification disorder, diffuse idiopathic skeletal hyperostosis (DISH), a very common hyperostotic disorder in Western countries (frequency of $25 \%$ in males and $15 \%$ in females at age more than 50 years), is a disorder known to be related to OPLL (Trojan et al. 1992; Weinfeld et al. 1997).

We have previously provided genetic linkage evidence of the genetic susceptibility of OPLL mapped to the HLA complex of chromosome 6 (Koga et al. 1998) by a nonparametric genetic linkage study with 91 affected sib-pairs with OPLL in Japan. We then extended our genetic study to search for molecular causality in candidate genes. Two genes in the region, collagen $\alpha 2$ (XI) (COL11A2) and retinoic $\mathrm{X}$ receptor $\beta(R X R B)$ were considered to be possible candidates for OPLL (Almasan et al. 1994; Nagata et al. 1995). The two genes were screened for the molecular variations by single-strand conformation polymorphism (SSCP) analysis. No SSCP variant was detected in the coding or promoter regions of $R X R B$ (Numasawa et al. 1999). In COL11A2, we initially identified 19 distinct single nucleotide polymorphisms (SNPs) through extensive SSCP screening that included the promoter region, 66 exons, and intron 1 (Koga et al. 1998). Of the 19 SNPs identified, significant association with OPLL was detected in 4 SNPs. Although the most significant association was observed 
with intron $6 \mathrm{SNP}$, the molecular causality is still unclear, because the 4 SNPs were in linkage disequilibrium with each other. Haplotype generation is a means of determining whether a specific allele mediates predisposition, especially when the causal variants involved in the disease have not been identified. Haplotype analysis, using the four SNPs, strongly indicated that $C O L 11 A 2$ plays an important role in the genetic etiology of OPLL (Koga et al. 1998). We have recently identified, by direct sequencing, a new SNP in exon 6 , a $\mathrm{G} \rightarrow$ A substitution at position 28 (nucleotide numbering is from the start of exon 6), which replaces glutamine at codon 272 by lysine. The exon 6 SNP was found to be in tight linkage disequilibrium with the intron 6 SNP both in OPLL patients and in controls. By increasing the number of the markers and the sample size, we found a gender-specific haplotype association of SNPs in the COL11A2 with OPLL, reported here.

\section{Subjects and methods}

\section{Subjects}

We examined a total of 161 unrelated Japanese OPLL patients $(83$ men; age [mean \pm SD], $60.65 \pm 10.44$ years, and 78 women; age, $59.14 \pm 9.02$ years) and 163 non-OPLL subjects (78 men; age, $74.06 \pm 6.47$ years, and 85 women; age, $76.22 \pm 6.51$ years), in Kagoshima, Japan, after obtaining their written informed consent. This study was approved by the Ethics Committee at the Faculty of Medicine, Kagoshima University. OPLL was diagnosed by the existence of ectopic ossification in the spinal ligaments, determined by radiographic examination of the cervical, thoracic, and lumbar spine; non-OPLL was determined by the absence of such ossification in the spinal ligaments. All the non-OPLL controls were over 65 years old, thus we excluded future patients.

\section{Allelic frequency and haplotype analysis}

Exon 6 SNP was identified by direct sequencing, in which nucleotide substitution at position 28 in exon 6 leads to glutamine-to-lysine substitution. The positions of the SNPs are described in the footnote of Table 1. The allele frequencies of the SNPs were determined by SSCP analysis (Orita et al. 1989; also described in Koga et al. 1998) for promoter -182 , exon 43, and exon 46 SNPs, and a mutagenically separated (MS)-polymerase chain reaction (PCR) method was used (Rust et al. 1993) for exon 6 and intron 6 SNPs. Information on primers and PCR conditions can be obtained from the authors. Estimation of differences in haplotype frequency between cases and controls was performed by the maximum-likelihood method, using a simplified version of the computer program GENEF (J-M Lalouel, University of Utah, unpublished). Procedures to generate the haplotype were described in full detail in Jeunemaitre et al. (1997). Briefly, two SNPs were chosen to generate the haplotype, followed by sequential inclusion of one SNP at a time. All haplotypes below a frequency of $1 / 4 \mathrm{~N}$, where $\mathrm{N}$ is the sample size, were automatically eliminated. Estimated haplotype frequencies were compared in patients and controls by the $\chi^{2}$ test of homogeneity, using a $2 \times 2$ contingency table, rather than the global test. Instead, probability values were corrected for multiple comparison by multiplying the $P$ value by the number of tests compared (Bonferroni adjustment). Pairwise linkage disequilibrium coefficiency was estimated and expressed as $\mathrm{D}^{\prime}=\mathrm{D} / \mathrm{D}_{\max }$ or $\mathrm{D} / \mathrm{D}_{\min }$, according to Thompson et al. (1988).

\section{Results}

Pairwise linkage disequilibrium

A significant association between OPLL patients and controls was observed in the newly identified exon $6(+28)$ SNP $(P=0.0012)$. Pairwise linkage disequilibrium between five SNPs in COL11A2 was estimated in controls (Table 2). Most of the SNPs were in tight linkage disequilibrium with each other. Notably, a strong linkage disequilibrium was retained $\left(\mathrm{D}^{\prime}=0.91\right)$ between the -182 promoter SNP and the exon $46 \mathrm{SNP}$ separated by $21.5 \mathrm{~kb}$. Almost identical linkage disequilibrium results were obtained in OPLL patients (data not shown).

Table 1. Estimated haplotype frequency in OPLL patients and non-OPLL subjects

\begin{tabular}{lllllll} 
Haplotype $^{\mathrm{a}}$ & $\begin{array}{l}\text { OPLL, } \\
n=161(322)\end{array}$ & $\begin{array}{l}\text { non-OPLL, } \\
n=163(326)\end{array}$ & $\chi^{2}(\mathrm{df}=1)$ & $P$ value & $P_{\text {corrected }}$ & OR (95\% CI) \\
\hline H1 (+++++) & 0.578 & 0.455 & 9.75 & 0.0018 & 0.009 & $1.614(1.325,1.943)$ \\
H2 $(+++-+)$ & 0.141 & 0.127 & 0.23 & 0.63 & $>1$ & $1.118(0.666,1.57)$ \\
H3 $(-++++)$ & 0.089 & 0.093 & 0.0056 & 0.94 & $>1$ & $1.059(0.524,1.594)$ \\
H4 $(-----)$ & 0.104 & 0.17 & 5.59 & 0.018 & 0.09 & $1.779(1.321,2.237)$ \\
Others $^{\text {b }}$ & 0.091 & 0.153 & 6.12 & 0.013 & 0.065 & \\
\hline
\end{tabular}

OPLL, Ossification of the posterior longitudinal ligament of the spine; OR, odds ratio; CI, confidence interval

${ }^{a}$ Plus (+) denotes common allele; minus $(-)$ denotes rare allele. Marker order from left to right: an $\mathrm{A} \rightarrow \mathrm{C}$ substitution at position -182 in the promoter region, a $\mathrm{G} \rightarrow$ A substitution in exon 6 (Glu272Lys), a $\mathrm{T} \rightarrow$ A substitution at position 636 in intron 6 (4 bases upstream from the start of exon 7), an $\mathrm{A} \rightarrow \mathrm{G}$ substitution in exon 43 (Pro1058Pro), and a $\mathrm{C} \rightarrow \mathrm{T}$ substitution in exon 46 (Pro1128Pro). $P$ values were corrected by the number of haplotypes observed (5 haplotypes), shown as $P_{\text {corrected }}$

${ }^{\mathrm{b}}$ See text for explanation 
Table 2. Pairwise linkage disequilibrium coefficients $\left(\mathrm{D}^{\prime}\right)$ between COL11A2 SNPs, estimated in Japanese controls

\begin{tabular}{lllll}
\hline & \multicolumn{2}{l}{$D^{\prime}$} & & \\
\cline { 2 - 5 } SNPs & -182 & Exon 6 $(+28)$ & Intron 6 $(-4)$ & Exon 43 $(+24)$ \\
\hline Exon 6 $(+28)$ & 0.89 & - & - & - \\
Intron 6 $(-4)$ & 0.73 & 0.75 & - & - \\
Exon 43 $(+24)$ & 0.46 & 0.65 & 0.80 & 1.00 \\
Exon 46 $(+18)$ & 0.91 & 0.78 & 0.81 & -
\end{tabular}

$D^{\prime}$ was estimated according to Thompson et al. (1988); 328 non-OPLL subjects were genotyped for all the loci

SNPs, Single nucleotide polymorphisms

Table 3. Gender-specific haplotype frequency in OPLL patients and non-OPLL subjects

\begin{tabular}{lccclll}
\hline Haplotype & OPLL & Non-OPLL & $\chi^{2}(\mathrm{df}=1)$ & $P$ value & $P_{\text {corrected }}$ & OR (95\% CI) \\
\hline Male: OPLL, $n=83$ & $(166)$; non-OPLL, $n=78$ & $(156)$ & & & \\
H1 $(+++++)$ & 0.639 & 0.436 & 13.3 & 0.0003 & 0.0015 & $2.29(1.84,2.73)$ \\
H2 $(+++-+)$ & 0.151 & 0.147 & 0.006 & 0.94 & $>1$ & $1.03(0.41,1.64)$ \\
H3 (-++++) & 0.078 & 0.103 & 0.58 & 0.45 & $>1$ & $1.35(0.58,2.11)$ \\
H4 (-- - - ) & 0.078 & 0.16 & 5.19 & 0.023 & 0.12 & $2.25(1.54,2.96)$ \\
Others & 0.054 & 0.154 & 8.68 & 0.0032 & 0.016 & \\
Female: OPLL, $n=78(156) ;$ non-OPLL, $n=85(170)$ & & & \\
H1 (+++++) & 0.564 & 0.494 & 1.6 & 0.21 & $>1$ & $1.33(0.89,1.76)$ \\
H2 (+++-+) & 0.115 & 0.094 & 0.39 & 0.53 & $>1$ & $1.26(0.54,1.97)$ \\
H3 (-++++) & 0.109 & 0.094 & 0.2 & 0.66 & $>1$ & $1.18(0.46,1.90)$ \\
H4 $(-----)$ & 0.147 & 0.182 & 0.72 & 0.46 & 2.3 & $1.29(0.70,1.88)$ \\
Others & 0.065 & 0.136 & 4.53 & 0.033 & 0.165 &
\end{tabular}

SNP order is the same as that shown in Table 1

+ , Wild-type allele; -, mutant allele

Estimation of haplotype frequency

We compared the estimated haplotype frequencies between OPLL patients and non-OPLL subjects with five SNPs in COL11A2. Four major haplotypes (H1-H4), together with four to five minor haplotypes, were generated, reflecting tight linkage disequilibrium (Table 1). Because the frequencies of the minor haplotypes were less than $5 \%$, they were combined and are shown as "others" in Table 1 (and Table 3). A significantly higher frequency of haplotype $\mathrm{H} 1$, comprising all the common alleles at each site, was observed in OPLL patients $\left(\chi^{2}=9.75\right.$; degrees of freedom $[\mathrm{df}]=1$; $P=0.0018$; corrected $P=0.009$ ), while a lower frequency of haplotype $\mathrm{H} 4$, comprising all the rarer alleles, was detected in the OPLL patients $\left(\chi^{2}=5.59\right.$; df $=1 ; P=0.018$; corrected $P=0.09$ ). The odds ratios for the risk for OPLL associated with the haplotype $\mathrm{H} 1$ and the haplotype $\mathrm{H} 4$ were 1.61 and 1.78 , respectively. To distinguish gender, we increased the proportion of female OPLL patients, and this may explain the less significant association than the association reported previously (Koga et al. 1998) although we increased the total sample size. The results of the genderspecific haplotype association study are presented separately (Table 3 ). In males, a highly significant increased frequency of haplotype $\mathrm{H} 1$ was observed in OPLL patients $\left(\chi^{2}=13.3 ; \mathrm{df}=1 ; P=0.0003\right.$; corrected $P=0.0015$; odds ratio $=2.29$ ) while a decreased frequency of $\mathrm{H} 4$ was also detected $\left(\chi^{2}=5.19 ; \mathrm{df}=1 ; P=0.023\right.$; corrected $P=0.12$; odds ratio $=2.25$ ). In contrast, no significant association in the major haplotypes $(\mathrm{H} 1-\mathrm{H} 4)$ was detected in females. Accordingly, there is a gender-specific association of the haplotype in COL11A2 with OPLL, and the haplotype H1 may be a predisposing allele, while the haplotype $\mathrm{H} 4$ could be a protective allele in males.

\section{Discussion}

We have found that the haplotypes of COL11A2 arising from the combination of five SNPs are associated with an increased risk of the development of OPLL, exclusively in males. No association was observed in females. OPLL is a complex trait, and complicated etiologies need to be considered for the understanding of the underlying pathogenesis, in which both genetic and environmental factors play mutual roles. OPLL is more frequent in males than in females, with a prevalence ratio of $2: 1$. OPLL commonly occurs at the cervical level in both males and females. Notably, thoracic ossification is frequently observed in females, but not in males. Our gender-specific association results suggest that genetic factors involved in COL11A2 play an important role in the etiology of OPLL in males, while sex hormones or other gender-specific factors may be more important than the genetic variation in COL11A2 in females. It is well established that estrogen plays a crucial role in the maintenance of bone mineral, because bone loss (osteoporosis) is frequently observed in postmenopausal 
women. Estrogen has a profound effect on bone development and remodeling (Oursler et al. 1991; Jilka et al. 1992). It should be emphasized that OPLL patients generally show a tendency of having high bone mineral density; therefore, they are in a hyperostotic state, regardless of age and sex. Although the genetic effect of COL11A2 could lead to hyperostosis in both genders, at an age of onset of around 50 years, most of the genetically high-risk women may escape manifestations of OPLL because of the tendency toward bone loss caused by estrogen decrease after menopause. This may explain, in part, the gender-specific genetic associations of COL11A2 and differences in disease prevalence between males and females.

The haplotype results of gender-specific association strongly implicate an important role of COL11A2 in the pathogenesis of OPLL in males. The evidence outlined above, in particular, the gender-specificity provides an important clue for understanding the molecular etiology of OPLL. In future, the functional impacts of the gene variation or the specific haplotype need to be elucidated, using modern experimental approaches, such as the application of genetically manipulated animal models.

Acknowledgments This study was supported by a Research Grant for Specific Diseases from the Ministry of Public Health and Welfare of Japan (S. Matsunaga; S.H.; and I.I.) and by the Japanese Ministry of Science, Education, Sports, and Culture (J.T; I.I). We are grateful to Dr. Jean-Marc Lalouel (University of Utah) for his valuable assistance in the haplotype analysis.

\section{References}

Almasan A, Mangelsdorf DJ, Ong ES, Wahl GM, Evans RM (1994) Chromosomal localization of the human retinoid $\mathrm{X}$ receptors. Genomics 20:397-403
Jeunemaitre X, Inoue I, Williams C, Charru A, Tichet J, Powers M, Sharma AM, Gimenez-Roqueplo AP, Hata A, Corvol P, Lalouel JM (1997) Haplotypes of angiotensinogen in essential hypertension. Am J Hum Genet 60:1448-1460

Jilka RL, Hangoc G, Girasole G, Passeri G, Williams DC, Abrams JS, Boyce B, Broxmeyer H, Manolagas SC (1992) Increased osteoclast development after estrogen loss: mediation by interleukin-6. Science 257:88-91

Koga H, Sakou T, Taketomi E, Hayashi K, Numasawa T, Harata S, Yone K, Matsunaga S, Otterud B, Inoue I, Leppert M (1998) Genetic mapping of ossification of the posterior longitudinal ligament of the spine. Am J Hum Genet 62:1460-1467

Matsunaga S, Sakou T (1997) Epidemiology of ossification of the posterior longitudinal ligament. In: Yonenobu K, Sakou T, Ono K (eds) Ossification of the posterior longitudinal ligament. Springer, Berlin Heidelberg New York Tokyo, pp 11-17

Nagata T, Weiss EH, Abe K, Kitagawa K, Abdo A, Yura-Kikuti Y, Seldin MF (1995) Physical mapping of the retinoid X receptor B gene in mouse and human. Immunogenetics 41:83-90

Numasawa T, Koga H, Ueyama K, Maeda S, Sakou T, Harata S, Leppert M, Inoue I (1999) Human retinoic X receptor $\beta$ : complete genomic sequence and mutation search for ossification of posterior longitudinal ligament of the spine. J Bone Miner Res 14:500-508

Orita M, Suzuki Y, Sekiya T, Hayashi K (1989) Rapid and sensitive detection of point mutations and DNA polymorphisms using the polymerase chain reaction. Genomics 5:874-879

Oursler MJ, Osdoby P, Pyfferoen J, Rigges BL, Spelsberg TC (1991) Avian osteoclasts as estrogen target cells. Proc Natl Acad Sci USA 88:6613-6617

Rust S, Funke H, Assmann G (1993) Mutagenically separated PCR (MS-PCR): a highly specific one-step procedure for easy mutation detection. Nucleic Acids Res 21:3623-3629

Terayama K (1989) Genetic studies on ossification of the posterior longitudinal ligament of the spine. Spine 14:1184-1191

Thompson EA, Deeb S, Walker D, Motulsky AG (1988) The detection of linkage disequilibrium between closely linked markers: RFLPs at the AI-CIII apolipoprotein genes. Am J Hum Genet 42:113124

Trojan DA, Pouchot J, Pokrupa R, Ford RM, Adamsbaum C, Hill RO, Esdaile JM (1992) Diagnosis and treatment of ossification of the posterior longitudinal ligament of the spine: report of eight cases and literature review. Am J Med 92:296-306

Weinfeld RM, Olson PN, Maki DD, Griffiths HJ (1997) The prevalence of diffuse idiopathic skeletal hyperostosis (DISH) in two large American Midwest metropolitan hospital populations. Skeletal Radiol 26:222-225 